\title{
Assessment of Rest Hypoxemia: Is there a Place for the 100\% Oxygen Test for Pathological Shunt Detection?
}

\author{
A Sow $^{1,2 *}$, J Finance ${ }^{1,3 *}$, A Boussuges ${ }^{1,4}$, S Delliaux ${ }^{1,5}$, J Guinde ${ }^{1}$, PA Thomas ${ }^{3,6}$, M Reynaud-Gaubert ${ }^{3,7}$ and \\ F Bregeon ${ }^{1,3 *}$ \\ ${ }^{1}$ Service des Explorations FonctionnellesRespiratoires Centre Hospitalo-Universitaire Nord, Pôlethoracique et cardio-vasculaire, France \\ ${ }^{2}$ Laboratoire de Physiologie et d'ExplorationsFonctionnelles, Faculté de Médecine, de Pharmacie et d'Odontologie de l'Université Cheikh \\ Anta Diop, Sénégal \\ ${ }^{3}$ Aix-Marseille Université, IRD, MEPHI, IHU Méditerranée Infection, France \\ ${ }^{4}$ Institut de Recherche Biomédicale des Armées (IRBA), Brétigny sur Orge
}

${ }^{5}$ INSERM, INRA, C2VN, Aix Marseille Université, France

${ }^{6}$ Department of Thoracic Surgery and Esophageal Diseases, Hôpital Nord, Assistance Publique-Hôpitaux de Marseille, Aix-Marseille University, France

${ }^{7}$ Service des Maladies RespiratoiresRares, Mucoviscidose, Pneumologie, Hôpital Nord, Assistance Publique-Hôpitaux de Marseille, AixMarseille University, France

*Corresponding author: F Bregeon, Service des Explorations FonctionnellesRespiratoires Centre Hospitalo-Universitaire Nord, Pôlethoracique et cardio-vasculaire, Aix-Marseille Université, IRD, MEPHI, IHU Méditerranée Infection, France

J Finance, Service des Explorations FonctionnellesRespiratoires Centre Hospitalo-Universitaire Nord, Pôlethoracique et cardiovasculaire, Aix-Marseille Université, IRD, MEPHI, IHU Méditerranée Infection, France

\section{ARTICLE INFO}

Received: 幽 August 13, 2020

Published: 幽 August 31, 2020

Citation: A Sow, J Finance, A Boussuges, S Delliaux, J Guinde, PA Thomas, M Reynaud-Gaubert, F Bregeon. Assessment of Rest Hypoxemia: Is there a Place for the 100\% Oxygen Test for Pathological Shunt Detection?. Biomed J Sci \& Tech Res 29(5)2020. BJSTR. MS.ID.004871.

Abbreviations: MHT: Hyperoxia Test; PFTs:Pulmonary Functional Tests; PFO: Patent Foramen Ovale; PH: Pulmonary Hypertension; PAM: Pulmonary Arterio-Venous Malformations; HT: Hyperoxia Test; FEV1: Forced Expiratory Volume in one second; FVC:Forced Vital Capacity; SVC: Slow Vital Capacity; TLC: Total Lung Capacity; RV: Residual Volumes; FRC: Functional Residual Capacity; SPAP: Systolic Pulmonary Arterial Pressure; MPAP: Mean Pulmonary Arterial Pressure; cTCD: Contrast Transcranial Doppler

\section{ABSTRACT}

Background: The Hyperoxia Test (HT) has been proposed to quantify the shunt fraction but it is often requested as a first-line diagnostic tool, without clear guidelines on its place in the strategy for diagnosing hypoxemia.

Objectives: To question the contribution of HT in managing patients with hypoxemia, taking into account comparison with morphological examinations.

Methods: We retrospectively went through on patient files referred to the Pulmonary Functional Test (PFT) laboratory from January 2016 to December 2019. Clinical, PFTs and echocardiographic data were collected.

Results: 53 cases were analyzed, HT showed a shunt above $7.5 \%$ (HT+ group) in $42(79 \%)$. Most of patients had a preexisting pulmonary disease. Rest $\mathrm{PaO}_{2}$ and DLCOc/VA significantly differed between HT- and HT+ Eighteen of the HT+ patients had morphological examinations, five had an anatomic abnormality. Estimated shunt fraction was negatively strongly correlated with rest $\mathrm{PaO}_{2}$ and DLCOc/VA. When compared to a predicted exploration strategy based on shunt value and PFTs abnormalities, the actual exploration strategy showed good concordance in HT- patients and some discrepancies in HT+ ones, but most of the time, there was a clinical reason to explain why a HT+ patients was no further investigated with echocardiography.

Conclusion: HT appeared as an easy-to-do diagnostic tool in hypoxemic patients that could help to rule out pathologic shunt and to screen those eligible for more complex specialized exploration.

Keywords: Hypoxemia; 100\% Oxygen Test;Shunt;Pulmonary Function; COPD; Foramen Ovale 


\section{Introduction}

Rest hypoxemia can be attributed to alteration of lung oxygenation function inducing abnormal distribution of the ventilation perfusion ratio (VA/Q) or disorders of the alveolarcapillary diffusion [1] leading to a shunt effect also called functional shunt. Nevertheless, when rest hypoxemia associates with normal or moderately altered Pulmonary Functional Tests (PFTs) results and normal imaging results of lung parenchyma, one should evoke an anatomic shunt. Even a shunt effect seems explained by abnormal PFTs, hypoxemia can lead to elevated lung arterial resistances, and thus right-sided pressures favoring Patent Foramen Ovale (PFO) thus anatomic and functional shunts can coexist and may interfere $[2,3]$ on blood oxygenation, as possibly observed in COPD patients, Pulmonary Hypertension (PH), in sleep apnea disorders or in patients with unexplained refractory hypoxemia.

In healthy subjects a physiologic shunt may not exceed 5\% of cardiac output at rest [4]. Higher values are considered as pathological and can be caused by PFO, other types of intracardiac defects, or less commonly to intra-pulmonary anatomical shunting via Pulmonary Arterio-Venous Malformations (PAM) [5] such as in the hepato-pulmonary syndrome [6] or the hereditary haemorrhagic telangiectasia disease. Despite intra-cardiac rightto-left shunting is one of the causes of hypoxemia [3], relationship between anatomical shunt, and hypoxemia remains unclear [79]. PFO closure can improve patients' outcome and quality of life [10-12]. The $100 \%$ oxygen inhalation test or Hyperoxia Test (HT) has been proposed since early 1960' to quantify the shunt fraction regardless its mechanism [13-15]. In 1968, S. T. Chiang proposed a formula to assess the shunt fraction after peripheral arteries blood gas analysis [16]. Visualization of PAM is ideally obtained using arteriography or injected computed tomography imaging. Concerning PFO, ultrasonic echocardiography is largely used. However, in hypoxemic patients, some limitations may be considered: contrast transesophageal echocardiography (cTEE) should not be tolerated well, contrast agents are contraindicated in case of severe emphysema [17] and trans-thoracic echocardiography (cTTE) can be thwarted because of low echogenicity as in obese or emphysematous patients [18]. Very few studies have focused on the contribution of the HT in hypoxemia diagnostic strategy. In hereditary hemorrhagic telangiectasia, Velthuis et al. have reported a low sensitivity of the HT in detecting abnormal shunt $(>5 \%)$ as compared to contrast cTTE as HT led to $51 \%$ false negative results [19]. Because high resolution imaging ultrasonographs are not always available for rapid routine examination, the HT could still have a place for respirologists in charge for hypoxemic patients. Here, we described our experience in a real-life retrospective descriptive study from a University-Hospital pulmonary function laboratory, trying to precise the contribution of the HT in hypoxemia diagnostic strategy.

\section{Methods \\ Study Design}

We investigated a retrospectively files of patients referred to the PFT laboratory of the University Hospital for a HT during January 2016 to December 2019. For this analysis, no additional procedure was added to standard of care. In the consultation ward, information about the analysis was available and the possibility was given to each patient to refuse the inclusion of self-medical data in the analysis. The collection and analysis of patients' data was approved by a local committee as per French legislation and is registered with the reference RGPD-APHM 20-251. Charts from patients of both gender in whom a HT was performed were included. In the absence of at least a spirometry test performed in the 7 days of the arterial blood gas samplings and in case of no confirmed hypoxemia, patients were were secondarily excluded. Study was approved by our Local Institutional Research Committee and conform the European General Regulation on Data Protection. Demographic parameters, main diagnosis, cardiovascular comorbidities, smoke history, underlying pulmonary disease including lung cancer, date and results of cTTE, cTEE and injected tomodensitometric imaging or arteriography when present were recorded.

\section{Functional and Blood Gas Measurements}

Forced Expiratory Volume in one second (FEV1), Forced Vital Capacity (FVC), Slow Vital Capacity (SVC), Total Lung Capacity (TLC), and Residual Volumes (RV) and Functional Residual Capacity (FRC) were recorded (MasterLab Jaeger plethysmograph, Bunnik, The Netherlands). Diffusing capacity for the lungs was measured by the single breath method using carbon monoxide (DLCO) with helium dilution [20]. Diffusing capacity for carbon monoxide per unit of alveolar volume (DLCO/VA or KCO) and it's correction by hemoglobin concentration (DLCOc/VA) were evaluated. Values were expressed in their physical unit and compared to the reference CECA 93 equations' values [21]. The lung functional were established according to ATS/ERS definitions [22]. The spirometrybased GOLD severity of COPD was graded from 1 (mild) to 4 (very severe) [23]. The severity of restrictive pattern and altered diffusing capacity of the lung for carbon monoxide were graded according to ATS/ERS classifications [22]. Hypoxemia was defined as partial arterial pressure of oxygen $\left(\mathrm{PaO}_{2}\right)$ value below predicted [24].

The HT was performed in the sitting position, the patient breathed 100\% oxygen from a Douglas bag via a closely fitting mask. Arterial blood sample was collected after 30-min exposition. The following Chiang's formula allowed to calculate the estimated shut including $\mathrm{PaCO}_{2}$ and actual Barometric Pressure (BP) [16]: shunt fraction $(\%)=\left(1.0557+0.0458 *\left(\mathrm{PAO}_{2}-\mathrm{PaO}_{2}\right)\right.$, where $\mathrm{PAO}_{2}=$ $\left[\left(\mathrm{BP}-\mathrm{PH}_{2} \mathrm{O}\right) * 1.00\right]-\mathrm{PaCO}_{2}$ (with PA was alveolar pressure). Given that literature data suggest a physiologic shunt between 5 and $8 \%$ when assessed by the HT, we arbitrarily fixed the threshold at 7.5 
$\%$ to consider abnormal shunt fraction. Thus shunt was estimated as pathologic (HT+ group when shunt $\geq 7.5 \%$ ) or physiologic (HTgroup when shunt $<7.5 \%$ ).

\section{Prediction of a Management Strategy from the PFTs Results and Shunt Value}

For the purpose of this study, before any clinical chart review, the shunt value was confronted to the PFTs results. An exploration management strategy was then predicted according to the following algorithm that we established on the basis of our common clinical sense and experience (Figure 1): Shunt value $<7.5 \%$ : complementary anatomical explorations were considered as OPTIONAL (not required since hypoxemia may be not shuntrelated).

Shunt between $7.5 \%$ and $20 \%$ :

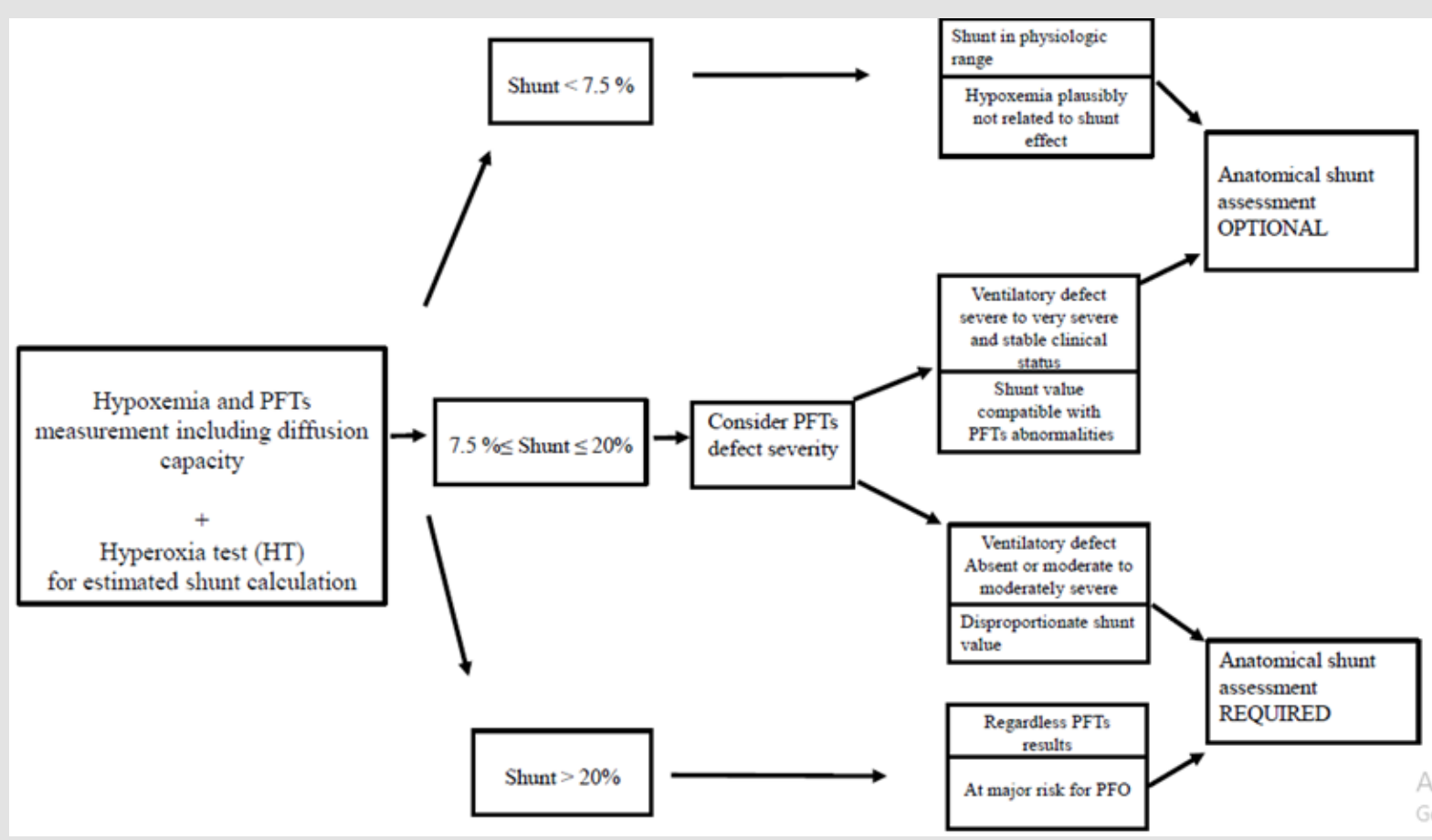

Figure 1: Algorithm for complementary explorations to assess for anatomical shunt after HT results.

1. when very severe PFTs abnormality coexisted, complementary explorations were considered as OPTIONAL (not mandatory to assess hypoxemia but could be done to detect a PFO if the patient was considered at risk).

2. when no abnormalities or moderately severe PFTs abnormalities were present, complementary explorations were considered as REQUIRED to detect possible anatomical shunting process.

Shunt $>20 \%$ : complementary explorations were considered as REQUIRED whatever the results of PFTs.

\section{Comparison of Predicted and Actual Managements}

Each shunt/PFT picture was allocated to the REQUIRED or OPTIONAL complementary exploration strategy by a person blinded of the actual strategy. The "actual" exploration strategy, i.e. that was really performed in the current practice after HT results, was then checked. Cases of discrepancies were then detailed.

\section{Complementary Investigations}

Intracardiac shunt was assessed by cTTE or cTEE. PAM was assessed using arteriography or injected computed tomography. PH was confirmed using a right heart catheterization. Data of Systolic Pulmonary Arterial Pressure (SPAP) And Mean Pulmonary Arterial Pressure (MPAP) were collected.

\section{Statistical Analysis}

We used free R software version 3.2.3. Normality distribution was checked using Shapiro's test. Results are expressed as means or medians \pm standard deviation. Student's t-test was used to compare normally distributed data, a Mann-Whitney's test for non-normally distributed ones and a chi-squared test for proportions applying the appropriate Yates correction when required. Correlations were assessed using Pearson test Figures were made with R version 3.2.3 and Graphpad Prism 5 software's. A p-value less than 0.05 was considered statistically significant. 


\section{Results}

\section{Study Population}

During the 48month period, 67 patients underwent HT, 63 of whom have recently been documented by PFT. Ten patients were secondarily excluded, 8 because of the absence of hypoxemia, one for failure of the HT and one patient was explored in a context of known PFO. Finally 53 patients were retained, DLCO data was missing in 7. Demographic characteristics are summarized in Table 1, most had a known underlying pulmonary disease and a lung functional defect. Only one patient had position-related rest hypoxemia. Clinical charts contained information on PH assessment for 17 patients. Values of pulmonary arterial pressure (SPAP and MPAP) were respectively $50.3 \pm 24.68 \mathrm{mmHg}$ and $42.25 \pm 16.03$ $\mathrm{mmHg}$.

Table 1: Demographic characteristics of the study population.

\begin{tabular}{|c|c|}
\hline Patients & N = 53 \\
\hline Age (mean year + SD; [min - max]) & $62.9 \pm 13.8 ;[21-91]$ \\
\hline BMI (mean kg/m² + SD; [min - max]) & $26.14 \pm 6.6 ;[15-49]$ \\
\hline \multicolumn{2}{|c|}{ Smoking history } \\
\hline Current smokers, n (\%) & $37(70 \%)$ \\
\hline Mean pack year +SD ; [min - max] & $32.44 \pm 19.7 ;[2-80]$ \\
\hline No or past smokers, n (\%) & $16(30 \%)$ \\
\hline Underlying pulmonary disease, n (\%) \\
\hline Asthma, n (\%) & $0(0 \%)$ \\
\hline CoPD, n (\%) & $21(40 \%)$ \\
\hline DILD, n (\%) & $6(15 \%)$ \\
\hline Lung cancer, n (\%) & $9(11 \%)$ \\
\hline Vasculitis, n (\%) & $9(17 \%)$ \\
\hline PH, n (\%) & $32(60 \%)$ \\
\hline Underlying cardiac disease \\
\hline Vascular comorbidity n (\%)
\end{tabular}

Note: BMI: body mass index; DILD: diffuse interstitial lung disease; $\mathrm{PH}$ : pulmonary hypertension.

\section{HT Results}

Of the 53 retained HT, 11 (21\%) had a shunt calculation < $7.5 \%$ (HT-). In the $42 \mathrm{HT}+$ results, 28 corresponded to a shunt between 7.5 and $20 \%, 14$ to a value $>20 \%$. The median value of estimated shunt was $17.06+6.54 \%$ in HT+ group versus $6.29+$ $2.03 \%$ in the HT- group, $\mathrm{p}<0.001$. For the 7 patients with missing DLCO values, all were HT+. In COPD patients ( $n=21$ or $39.6 \%$ of the cohort), the rate of HT+ was high $(17 / 21$ or $81 \%)$ and shunt values reached the highest values (above 25\%). Two of HT+ COPD patients had also a lung cancer, their shunt values were $30.21 \%$ and $26.37 \%$ respectively. Four patients had fully normal PFTs (2 in the HT- group and 2 in the HT+ group). As expected, HT+ group showed significantly lower rest $\mathrm{PaO}_{2}$ value than HT- group (Table 2). The DLCOc/VA percent predicted value was markedly and significantly lower in HT+ compared to HT- patients. Also, the estimated shunt was significantly lower in patients with decreased DLCOc/VA below $80 \%$ predicted compared to patients with fully normal values $(16.21 \%+7.88$ vs $10.86+10.25 \%, \mathrm{p}=0.01)$.

Table 2: PFT results in patients with an estimated shunt as physiologic (HT-) or pathologic - HT+), in mean + SD, [min $\max ]$.

\begin{tabular}{|c|c|c|c|}
\hline & HT+ & HT- & \multirow{2}{*}{$\mathbf{p}$} \\
\hline & $\mathrm{N}=42$ & $N=11$ & \\
\hline \multirow{2}{*}{ FEV1, L } & $1.93 \pm 0.83$ & $2.17 \pm 0.7$ & \multirow{2}{*}{ NS } \\
\hline & {$[0.5-3.6]$} & {$[0.94-3.27]$} & \\
\hline \multirow{2}{*}{ FEV1, \% predicted } & $70.52 \pm 24.85$ & $71.6 \pm 23.8$ & \multirow{2}{*}{ NS } \\
\hline & [19.7 - 110.03] & {$[26.74-110.8]$} & \\
\hline \multirow{2}{*}{ FEV1/FVC, \% } & $70.4 \pm 17.02$ & $74.72 \pm 14.27$ & \multirow{2}{*}{ NS } \\
\hline & [27.38 - 100.00] & [51.82 - 94.32] & \\
\hline \multirow{2}{*}{ DLCO, \% predicted } & $41.8 \pm 27.01$ & $50.1 \pm 20.6$ & \multirow{2}{*}{ NS } \\
\hline & {$[12.3-113.5]$} & {$[17.46-86.2]$} & \\
\hline \multirow{2}{*}{$\begin{array}{c}\text { DLCOc/VA, \% } \\
\text { predicted }\end{array}$} & $49.23 \pm 25.22$ & $66.81 \pm 26.77$ & \multirow{2}{*}{$<0.05$} \\
\hline & [15.5 - 120.39] & [28.23 - 114.49] & \\
\hline \multirow{2}{*}{ TLC, \% predicted } & $96.31 \pm 25.72$ & $93.00 \pm 20.11$ & \multirow{2}{*}{ NS } \\
\hline & [36.52 - 145.06] & [53.45 - 128.99] & \\
\hline \multirow{2}{*}{$\mathrm{PaO}_{2}$ rest, $\mathrm{mmHg}$} & $57.25 \pm 9.36$ & $66.32 \pm 7.95$ & \multirow{2}{*}{$<0,01$} \\
\hline & {$[35-72]$} & {$[53-78.11]$} & \\
\hline \multirow{2}{*}{$\mathrm{PaCO}_{2}$ rest, mmHg } & $34.35 \pm 5.74$ & $34.91 \pm 5.80$ & \multirow{2}{*}{ NS } \\
\hline & {$[24.2-48.5]$} & {$[25.2-43.8]$} & \\
\hline
\end{tabular}

Note: FEV1: Forced expiratory volume in one second

FVC: Forced vital capacity

DLCO: Diffusing capacity for the lungs measured using carbon monoxide

DLCOc/VA: Diffusing capacity for carbon monoxide per unit of alveolar volume corrected by the haemoglobin concentration

TLC: Total lung capacity

NS: Not significant

DLCO, DLCOc/VA, rest $\mathrm{PaO}_{2}$ and $\mathrm{SaO}_{2}$ were negatively and significantly correlated with the estimated shunt value as shown by matrix correlation of Figure $2 \mathrm{~A}$. The strongest correlation observed with the shunt value concerned DLCOc/VA and can be illustrated by the equation estimated shunt $=-3.146 * \mathrm{DLCOc} / \mathrm{VA}+22.12 ; \mathrm{p}=$ 0.001 and $\mathrm{R}^{2}=0.22$ (Figure $2 \mathrm{~B}$ ). 


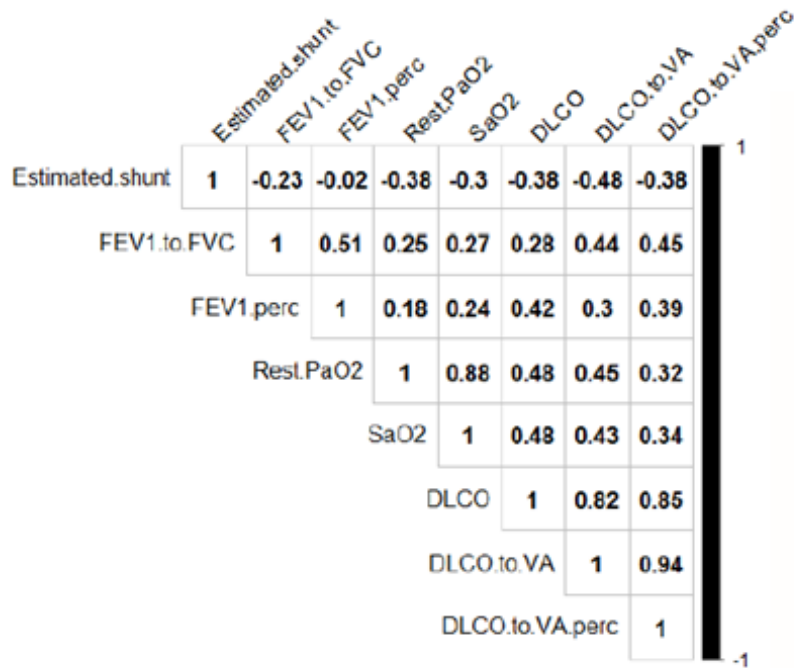

A

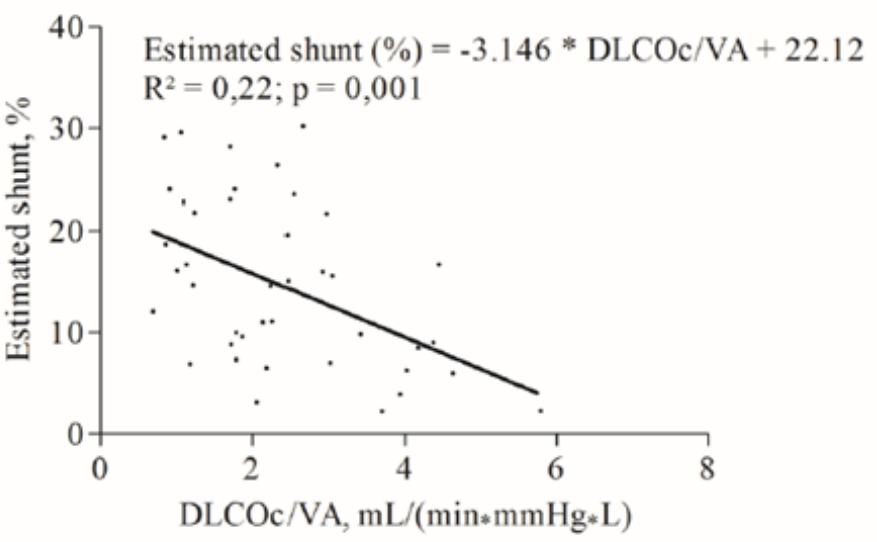

B

Figure 2: Correlation between PFTs parameters and rest arterial oxygenation with estimated shunt fraction. A: Correlation matrix. B: Linear regression and Pearson correlation test results between estimated shunt and DLCOc/VA.

\section{Complementary Investigations in HT+ Patients}

Of the $42 \mathrm{HT}+$ patients, 18 were further explored, none of the HT- group were. Six of these 18 explored patients were diagnosed to had an anatomical abnormality compatible with a shunt as attested by PAM in one case, a hypervascularized voluminous tumor in 1 case and a PFO in four cases, 2/4 PFO undergone further PFO closure, both had underlying COPD and were listed on a transplantation program. Interestingly, hypoxemia did not appear more severe in HT+ patients who had complementary explorations than HT+ patients who had not. The lung volumes tended to be higher in explored patients, especially total lung capacity (Table 3). In the 18 explored patients, FEV1 and FEV1 / FVC tended to be lower in those with anatomical defects than in those with functional shunt (Table 4).

Table 3: PFTs results in explored and not explored groups by echocardiography, in mean \pm SD, [Min - Max].

\begin{tabular}{|c|c|c|c|}
\hline & Explored group & Not explored group & \multirow{2}{*}{$\mathbf{p}$} \\
\hline & $N=18$ & $N=24$ & \\
\hline \multirow{2}{*}{ FEV1, L } & $2.2 \pm 0.92$ & $1.71 \pm 0.66$ & \multirow{2}{*}{ NS } \\
\hline & {$[0.5-3.44]$} & {$[0.87-3.6]$} & \\
\hline \multirow{2}{*}{ FEV1, \% predicted } & $78.35 \pm 28.1$ & $65.75 \pm 21.3$ & \multirow{2}{*}{ NS } \\
\hline & [19.7-109.5] & {$[27.68-110.03]$} & \\
\hline \multirow{2}{*}{ FEV1/FVC, \% } & $66.47 \pm 17.68$ & $74.14 \pm 16.1$ & \multirow{2}{*}{ NS } \\
\hline & [27.38 - 86.99] & [42.92 - 99.9] & \\
\hline \multirow{2}{*}{ DLCO, \% predicted } & $48.12 \pm 31.6$ & $37.08 \pm 22.7$ & \multirow{2}{*}{ NS } \\
\hline & [13.2-113.5] & {$[12.3-94.47]$} & \\
\hline \multirow{2}{*}{ DLCOc/VA, \% predicted } & $56.48 \pm 32.52$ & $48.89 \pm 19.7$ & \multirow{2}{*}{ NS } \\
\hline & {$[16.9-120.4]$} & {$[15.5-90.59]$} & \\
\hline \multirow{2}{*}{ TLC, \% predicted } & $110.2 \pm 22.4$ & $85.14 \pm 22.3$ & \multirow{2}{*}{0.001} \\
\hline & {$[60.87-145.06]$} & [36.52-133] & \\
\hline \multirow{2}{*}{ Rest.PaO ${ }^{2}, \mathrm{mmHg}$} & $57.51 \pm 9.82$ & $58.01 \pm 9.7$ & \multirow{2}{*}{ NS } \\
\hline & {$[36.1-73.9]$} & {$[35-72]$} & \\
\hline \multirow{2}{*}{ Rest.PaCO ${ }^{2}, \mathrm{mmHg}$} & $33.56 \pm 5.68$ & $35.1 \pm 5.7$ & \multirow{2}{*}{ NS } \\
\hline & {$[24.2-48.5]$} & {$[25.5-47]$} & \\
\hline
\end{tabular}


Note: FEV1: Forced expiratory volume in one second

FVC: Forced vital capacity

DLCO: Diffusing capacity for the lungs measured using carbon monoxide

DLCOc/VA: Diffusing capacity for carbon monoxide per unit of alveolar volume corrected by the haemoglobin concentration

TLC: Total lung capacity

NS: Not significant

Table 4: PFT result in subgroups of patients whether an anatomical shunt was evidenced or not evidence, in mean \pm SD, [Min - Max].

\begin{tabular}{|c|c|c|}
\hline & Anatomical defect & Not evidence of anatomic defect \\
\hline & $N=6$ & $\mathrm{~N}=12$ \\
\hline \multirow{2}{*}{ FEV1, L } & $2.04 \pm 1.02$ & $2.3 \pm 0.9$ \\
\hline & {$[0.5-3.44]$} & {$[0.54-3.26]$} \\
\hline \multirow{2}{*}{ FEV1, \% predicted } & $78.7 \pm 29.3$ & $78.2 \pm 28.8$ \\
\hline & {$[23.15-102.5]$} & [19.7-109.5] \\
\hline \multirow{2}{*}{ FEV1/FVC, \% } & $63.6 \pm 16.5$ & $67.93 \pm 18.8$ \\
\hline & [34.5 - 79.13] & [27.38 - 86.99] \\
\hline \multirow{2}{*}{ DLCO, \% predicted } & $62.38 \pm 39.3$ & $41 \pm 26.39$ \\
\hline & {$[20.39-113.5]$} & {$[13.2-90.3]$} \\
\hline \multirow{2}{*}{ DLCOc/VA, \% predicted } & $63.27 \pm 37.8$ & $52.7 \pm 31$ \\
\hline & {$[22.34-120.4]$} & {$[16.9-107.16]$} \\
\hline \multirow{2}{*}{ TLC, \% predicted } & $121.9 \pm 14.3$ & $104.4 \pm 24$ \\
\hline & {$[104.8-142.8]$} & {$[60.87-145.06]$} \\
\hline \multirow{2}{*}{ Rest.PaO ${ }^{2}, \mathrm{mmHg}$} & $55.1 \pm 12.25$ & $58.72 \pm 8.72$ \\
\hline & {$[36.1-71.81]$} & {$[44.6-73.9]$} \\
\hline \multirow{2}{*}{ Rest.PaCO ${ }^{2}, \mathrm{mmHg}$} & $33.7 \pm 8.3$ & $33.49 \pm 4.31$ \\
\hline & [24.2- 48.5] & {$[29.2-44.4]$} \\
\hline
\end{tabular}

Note: FEV1: Forced expiratory volume in one second

FVC: Forced vital capacity

DLCO: Diffusing capacity for the lungs measured using carbon monoxide

DLCOc/VA: Diffusing capacity for carbon monoxide per unit of alveolar volume corrected by the haemoglobin concentration

TLC: Total lung capacity

NS: Not significant

\section{Comparison of Predictive and Actual Management Strategies}

Analysis between the predicted and the actual exploration strategies showed a $100 \%$ concordance in the 11 HT- patients as none of the patients had additional exploration for anatomic shunt detection. For the 28 patients with shunt from 7.5 to $20 \%$, our algorithm indicated complementary exploration as OPTIONAL in 17 and as REQUIRED in 11. Actual exploration management was CTTE in 3 of the 17 OPTIONAL pictures leading to diagnose a moderate PFO in one case with no decision of closure, and in 6 of the 11 REQUIRED pictures. Two cases of abnormalities compatible with shunt were documented in the 11 REQUIRED cases: 1 hypervascularized lung cancerous tumor and 1 hepatopulmonary syndrome. Among the 5 unexplored patients from the REQUIRED arm, one had a BMI above $30 \mathrm{~kg} / \mathrm{m}^{2}$ and fully normal PFTs results and was further investigated for sleep apnea disorder,
1 was emphysematous and required high oxygenation supply, and the 3 others had no evidence of contraindication for anatomical exploration.

In the $14 \mathrm{HT}+$ patients with a shunt $>20 \%$, 9 had complementary explorations leading to diagnose an anatomical shunt in 3 (3 PFO). All the 5 non-explored patients had severe PFTs abnormalities. In details, one unexplored patient was aged 91 year, so the relevance of incrementing investigations was probably estimated as too low; one had severe emphysema and severe hypoxemia so both CTTE and CTEE were not feasible; one had severe obesity and presented unstable coronary artery disease; the two remaining patients had severely altered diffusing capacity but moderate hypoxemia at 8 and $8.13 \mathrm{KPa}$. Their respective shunt values were 24 and $26.4 \%$, no further investigation was found in their clinical charts. Taken together, complementary explorations were indicated as REQUIRED for 25 patients of whom 15 were actually explored (60\%), 5 had 
plausible contraindication and 5 were unexplored with no clear clinical contraindications.

\section{Discussion}

This real-life study on current practice of HT in addition to PFTs showed that 1) most of the suspected cases (79\%) of elevated shunt fraction were confirmed by HT at the retained threshold of 7.5\% 2) DLCOc/VA was the most discriminant to decipher between elevated and normal shunt values 3 ) the patients in whom a PFO detection was plausibly indicated, $25 \%$ had no assessment for anatomical shunt detection. Easy to use in a PFTs laboratory, HT can be more rapidly scheduled than specialized ultrasonic examinations. In our center, HT was often requested in first-line as a screening test. Patients were mainly COPD and DILD, some being planned for lung transplantation surgery. To decrease false positive results, the threshold is usually elevated above the physiologic value of $5 \%$ for the shunt. Here, we arbitrarily fixed at $7.5 \%$ the threshold.

The $100 \%$ concordance between predicted and actual exploration strategies in the HT- group suggests that HT was taken into account by physician in charge of the patient with high confidence to rule-out pathologic shunt. Between 7.5 and 20\%, interpretation of a shunt value appeared more variable. In the 17 patients in whom complementary explorations were considered as OPTIONAL according to our algorithm, 3 were explored, indicating that when severe PFTs abnormalities are present, there was a good agreement between predicted and actual exploration strategies. The presence of a PFO in one of these cases did not influence his clinical management. It is plausible that PFO detection in a patient with shunt value $<20 \%$, was only a reflect of the high incidence of PFO in human. PFO is a remnant of the fetal circulation documented in $25 \%$ to $40 \%$ of general population $[25,26]$. The clinical management of some of our cases with shunt values $>20 \%$ would require special attention, only 9 of 14 being explored. Even considering that one 91-y old patient would not benefit incremental explorations and considering patients with severe emphysema and hypoxemia or unstable coronary disease as anechogenic on cTTE or unable to tolerate cTEE, it remained only 2 patients at risk for PFO who were not explored. Their respective shunt was 24 and $26.4 \%$.

Our 33\% rate of explored COPD patients who were confirmed to have a PFO agrees with that obtained in autopsy studies in the general population [25] or in patients with respiratory impairment [9]. Interestingly, hypoxia was not more profound in HT+ patients actually explored with cTTE or CTEE, than in non-explored ones suggesting that the severity of hypoxemia was not the main factor influencing further investigation demand. On the other hand, explored patients had better lung volumes, suggesting that the discrepancies between severity of hypoxemia and relatively good forced and total lung volumes was the motivation for further investigations. Recent study has shown that PFO in the COPD patient is as common as in the general population. However, when a
PFO is present in a COPD patient, it is of more severe grade than for healthy subjects [9]. Here, FEV1 and FEV1/FVC tended to be lower in the anatomical shunt sub-group comforting previously published data in severe COPD patients. Both CTEE and cTTE allow arguing for the cardiac or extra-cardiac origin of anatomic shunting [27]. Apart from cardiac ultrasonic examinations, Contrast Transcranial Doppler (CTCD) is now considered the most sensitive and specific method for shunt detection [28-30]. As compared to cTEE and cTTE, cTCD fails to distinguish intra-pulmonary and intra-cardiac shunts and should be reserved at the screening step [28] cTCD was not available at the time of the study in our center and was not reported in this series.

Because most of the explored patients were previously known has having lung function alteration and/or lung disease, it is unclear what precisely decided the physician in charge to request for a shunt detection except in patients with normal or moderately altered PFTs. In one case, the sudden aggravation in oxygen requirement in a functionally stable COPD patient appeared the main reason. In two lung transplant candidates, the shunt assessment was required as part of the medical plan. HT cannot decipher between functional and anatomic shunt but offers the advantage of a precise quantification of the shunt even if not denied of false positive or false negative results. DLCOc/VA values was the only PFTs parameter that significantly differed between HT+ and HT- groups, and DLCOc/VA was significantly correlated to the shunt value. Our study did not clarify whether PFTs abnormalities or shunt value could identify patients with anatomical shunt, mainly PFO. Interestingly restrictive as well as obstructive defects were observed in patients presenting PFO.

\section{Conclusion}

The benefits of the HT at first-line appear clear when it allows ruling out a shunt in hypoxemic patients. When a pathological shunt is documented, some patients remain unexplored but most of the time because of plausible clinical reason Exploration tools including HT and PFTs, especially DLCOc/VA value, should help to screen patients with pathological shunt.

\section{Acknowledgement}

Statement of Ethics. For this analysis, no additional procedure was added to standard of care. In the consultation ward, information about the analysis was available and the possibility was given to each patient to refuse the inclusion of self medical data in the analysis. The collection and analysis of patients' data was approved by a local committee as per French legislation and is registered with the reference RGPD-APHM 20-251.

\section{Conflict of Interest Statement}

None of the co-author had any financial interests or nonfinancial relationships in relation with the manuscript at any stage of its preparation and redaction. 


\section{Funding Sources}

There was no funding.

\section{Author Contribution}

AS, JF and FB conceived the study, collected data and wrote the manuscript, SD contributed to statistics, JG contributed to data collection, all co-authors reviewed the MS.

\section{References}

1. Rodriguez-Roisin R, Drakulovic M, Rodriguez DA, Roca J, Barbera JA, et al. (2009) Ventilation-perfusion imbalance and chronic obstructive pulmonary disease staging severity. Journal of applied physiology (Bethesda, Md: 1985) 106(6): 1902-1908.

2. Lovering AT, Elliott JE, Beasley KM, Laurie SS (2010) Pulmonary pathways and mechanisms regulating transpulmonary shunting into the general circulation: an update. Injury 41: S16-S23.

3. Agrawal A, Palkar A, Talwar A (2017) The multiple dimensions of Platypnea-Orthodeoxia syndrome: A review. Respiratory medicine 129: 31-38.

4. Wagner PD (2015) The physiological basis of pulmonary gas exchange: implications for clinical interpretation of arterial blood gases. The European respiratory journal 45(1): 227-243.

5. Miller WC, Heard JG, Unger KM (1984) Enlarged pulmonary arteriovenous vessels in COPD. Another possible mechanism of hypoxemia. Chest 86(5): 704-706.

6. Koch DG, Fallon MB (2014) Hepatopulmonary syndrome. Clinics in liver disease 18(2): 407-420.

7. Juliard JM, Aubry P, Ducrocq G, Lepage L, Brochet E (2009) Should we close hypoxaemic patent foramen ovale and interatrial shunts on a systematic basis? Archives of cardiovascular diseases 102(11): 739-741.

8. Mojadidi MK, Ruiz JC, Chertoff J, Zaman MO, Elgendy IY, et al. (2019) Patent Foramen Ovale and Hypoxemia. Cardiology in review 27(1): 3440

9. Shaikh ZF, Kelly JL, Shrikrishna D, Villa M, Mullen MJ, et al. (2014) Patent foramen ovale is not associated with hypoxemia in severe chronic obstructive pulmonary disease and does not impair exercise performance. American journal of respiratory and critical care medicine 189(5): 540-547.

10. Mojadidi MK, Gevorgyan R, Noureddin N, Tobis JM (2015) The effect of patent foramen ovale closure in patients with platypnea-orthodeoxia syndrome. Catheterization and cardiovascular interventions: official journal of the Society for Cardiac Angiography \& Interventions 86(4): 701-707.

11. Silver B, Greenbaum A, Mc Carthy S (2007) Improvement in sleep apnea associated with closure of a patent foramen ovale. Journal of clinical sleep medicine: JCSM: official publication of the American Academy of Sleep Medicine 3(3): 295-296.

12. Meier B (2012) Some air for closure of the patent foramen ovale. JACC Cardiovascular interventions 5(4): 420-421.

13. Ravin MB, Epstein RM, Malm JR (1965) Contribution of thebesian veins to the physiologic shunt in anesthetized man. Journal of applied physiology 20(6): 1148-1152.

14. Shapiro AR, Virgilio RW, Peters RM (1977) Interpretation of alveolararterial oxygen tension difference. Surgery, gynecology \& obstetrics 144(4): 547-552.

15. Whyte MK, Peters AM, Hughes JM, Henderson BL, Bellingan GJ, et al. (1992) Quantification of right to left shunt at rest and during exercise in patients with pulmonary arteriovenous malformations. Thorax 47(10): 790-796.

16. Chiang ST (1968) A nomogram for venous shunt (Q்s/Q̇t) calculation. Thorax 23(5): 563-565.

17. Modonesi E, Balbi M, Bezante GP (2010) Limitations and potential clinical application on contrast echocardiography. Current cardiology reviews 6(1): 24-30.

18. Yue L, Zhai YN, Wei LQ (2014) Which technique is better for detection of right-to-left shunt in patients with patent foramen ovale: comparing contrast transthoracic echocardiography with contrast transesophageal echocardiography. Echocardiography (Mount Kisco, NY) 31(9): 10501055 .

19. Velthuis S, Vorselaars VM, Westermann CJ, Snijder RJ, Mager JJ, et al. (2015) Pulmonary shunt fraction measurement compared to contrast echocardiography in hereditary haemorrhagic telangiectasia patients: time to abandon the $100 \%$ oxygen method? Respiration; international review of thoracic diseases 89(2): 112-118.

20. Macintyre N, Crapo RO, Viegi G, Johnson DC, Van der Grinten CPM, et al. (2005) Standardisation of the single-breath determination of carbon monoxide uptake in the lung. The European respiratory journal 26(4): 720-735.

21. Quanjer PH, Tammeling GJ, Cotes JE, Pedersen OF, Peslin R, et al. (1993) Lung volumes and forced ventilatory flows. Report Working Party Standardization of Lung Function Tests, European Community for Steel and Coal. Official Statement of the European Respiratory Society. The European respiratory journal Supplement 16: 5-40.

22. Pellegrino R, Viegi G, Brusasco V, Crapo RO, Burgos F, et al. (2005) Interpretative strategies for lung function tests. The European respiratory journal 26(5): 948-968.

23. Vogelmeier CF, Criner GJ, Martinez FJ, Anzueto A, Barnes PJ, et al. (2017) Global Strategy for the Diagnosis, Management, and Prevention of Chronic Obstructive Lung Disease 2017 Report. GOLD Executive Summary. American journal of respiratory and critical care medicine 195(5): 557-582

24. Crapo RO, Jensen RL, Hegewald M, Tashkin DP (1999) Arterial blood gas reference values for sea level and an altitude of 1,400 meters. American journal of respiratory and critical care medicine 160(5 Pt 1): 1525-1531.

25. Hagen PT, Scholz DG, Edwards WD (1984) Incidence and size of patent foramen ovale during the first 10 decades of life: An autopsy study of 965 normal hearts. Mayo Clinic proceedings 59(1): 17-20.

26. Woods TD, Harmann L, Purath T, Ramamuthy S, Subramanian S, et al. (2010) Small- and moderate-size right-to-left shunts identified by saline contrast echocardiography are normal and unrelated to migraine headache. Chest 138(2): 264-269.

27. Mas JL, Arquizan C, Lamy C, Zuber M, Cabanes L, et al. (2001) Recurrent cerebrovascular events associated with patent foramen ovale, atrial septal aneurysm, or both. The New England journal of medicine 345(24): 1740-1746

28. Mahmoud AN, Elgendy IY, Agarwal N, Tobis JM, Mojadidi MK (2017) Identification and Quantification of Patent Foramen Ovale-Mediated Shunts: Echocardiography and Transcranial Doppler. Interventional cardiology clinics 6(4): 495-504.

29. Mojadidi MK, Roberts SC, Winoker JS, Romero J, Goodman-Meza D, et al. (2014) Accuracy of transcranial Doppler for the diagnosis of intracardiac right-to-left shunt: a bivariate meta-analysis of prospective studies. JACC Cardiovasc Imaging 7(3): 236-250.

30. Devuyst G, Piechowski-Jozwiak B, Karapanayiotides T, Fitting JW, Kemeny V, et al. (2004) Controlled contrast transcranial Doppler and arterial blood gas analysis to quantify shunt through patent foramen ovale. Stroke 35(4): 859-863. 
ISSN: 2574-1241

DOI: $10.26717 /$ BJSTR.2020.29.004871

A Sow, J Finance, F Bregeon. Biomed J Sci \& Tech Res

(c) (9) This work is licensed under Creative Commons Attribution 4.0 License

Submission Link: https://biomedres.us/submit-manuscript.php

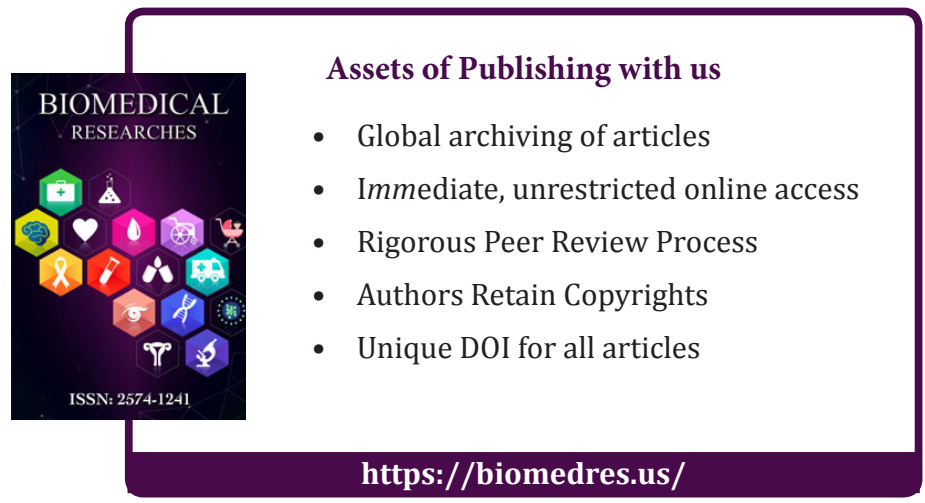

